\title{
Favourable outcome after peripartum cardiomyopathy: a ten-year study on peripartum cardiomyopathy in a university hospital
}

Kok-Han Chee, MBBS, FESC

INTRODUCTION Peripartum cardiomyopathy (PPCM) is an uncommon form of congestive heart failure, affecting obstetric patients around the time of delivery. The epidemiology of PPCM is infrequently reported. This study was undertaken to define the prevalence, presentation and outcome of PPCM among women giving birth in a teaching hospital in Malaysia.

METHODS A retrospective case record analysis was conducted on all patients admitted and diagnosed with PPCM at the University Malaya Medical Centre, Kuala Lumpur, Malaysia, from 1 January 2000 to 31 December 2009. All deliveries were undertaken in the same hospital.

RESULTS A total of 12 patients were diagnosed with PPCM during the ten-year study period. The prevalence of PPCM was 2.48 in 100,000 (1 in 40,322) live births. Nine women were diagnosed with PPCM within five months of delivery. Three women had twin pregnancies. There was one death in the group (mortality rate 8.3\%). The mean left ventricular ejection fraction at the time of diagnosis was $28.9 \% \pm 8.5 \%$ (range $15 \%-40 \%$ ). Following the index event, left ventricular function normalised in six of the nine patients $(66.7 \%)$ who underwent subsequent echocardiography one year later. All patients were treated with standard heart failure therapy. Two patients with normalised left ventricular function had subsequent pregnancies - one pregnancy was terminated at seven weeks and the other patient delivered uneventfully at full term. CONCLUSION PPCM is uncommon. The outcome in our series was favourable, with $66.7 \%$ of patients with PPCM recovering their left ventricular function. The mortality rate was $8.3 \%$.

Keywords: epidemiology, heart failure, peripartum cardiomyopathy, pregnancy

Singapore Med J 2013; 54(1): 28-31

\section{INTRODUCTION}

Peripartum cardiomyopathy (PPCM) is an uncommon form of congestive heart failure complicating pregnancy. Virchow first observed the occurrence of heart failure in pregnancy in the 1890s. However, the association between cardiomyopathy and pregnancy was only reported in 1937 by Gouley et al. ${ }^{(1)}$ According to the literature, PPCM occurs in one of every 3,000-15,000 pregnancies, with its incidence being higher in Africa. ${ }^{(2,3)}$ The present study builds on a previous report of PPCM in the Asian region by Chee and Azman. ${ }^{(4)}$

In 1971, Demakis and Rahimtoola ${ }^{(5)}$ established the criteria for the diagnosis of PPCM as: (a) heart failure must manifest in the last month of pregnancy or within five months of delivery; and (b) a diagnosis of PPCM should be made only when other aetiologies for heart failure cannot be found. The diagnostic criteria for PPCM were subsequently refined in 2000 by the National Heart, Lung and Blood Institute (NHLBI) and the Office of Rare Diseases of the National Institutes of Health (NIH) Workshop on PPCM. ${ }^{(6)}$ The definition of PPCM now includes: (a) development of cardiac failure in the last month of pregnancy or within five months of delivery; (b) absence of an identifiable cause of cardiac failure; (c) absence of recognisable heart disease before the last month of pregnancy; and (d) left ventricular systolic dysfunction with left ventricular ejection fraction (LVEF) $<45 \%$ by echocardiography.
The natural history of PPCM is poorly characterised. This is likely due to the limited number of patients being reported and the variations in the diagnostic criteria being used by various authors, along with the degree to which these authors pursue exclusion of other heart disease. Safirstein et al, in a prospective registry of patients with PPCM recruited via the Internet, found that the presence of gestational hypertension, LVEF > 35\% at diagnosis, breastfeeding and postpartum diagnosis were all significantly associated with recovery of systolic function in the affected women. ${ }^{(7)}$ The prognosis of PPCM is also quite variable. Subsequent pregnancy in women with a history of PPCM is associated with a significant decrease in left ventricular function and can result in clinical deterioration, even death. This is especially true for those with persistent depressed left ventricular function. ${ }^{(8)}$ The risk, however, seems to be lower among patients who have regained normal ventricular function. ${ }^{(9)}$ Maternal mortality rates in the United States have been estimated to be $25 \%-50 \% .{ }^{(10)}$ Death in the reported series have usually been due to progressive left ventricular failure and associated complications, including arrhythmia and thromboembolism. ${ }^{(11)}$

The epidemiology of PPCM in Asia is largely unknown. Long-term prevalence studies on such patients are scarce in the literature as well. In this retrospective case record analysis, the 


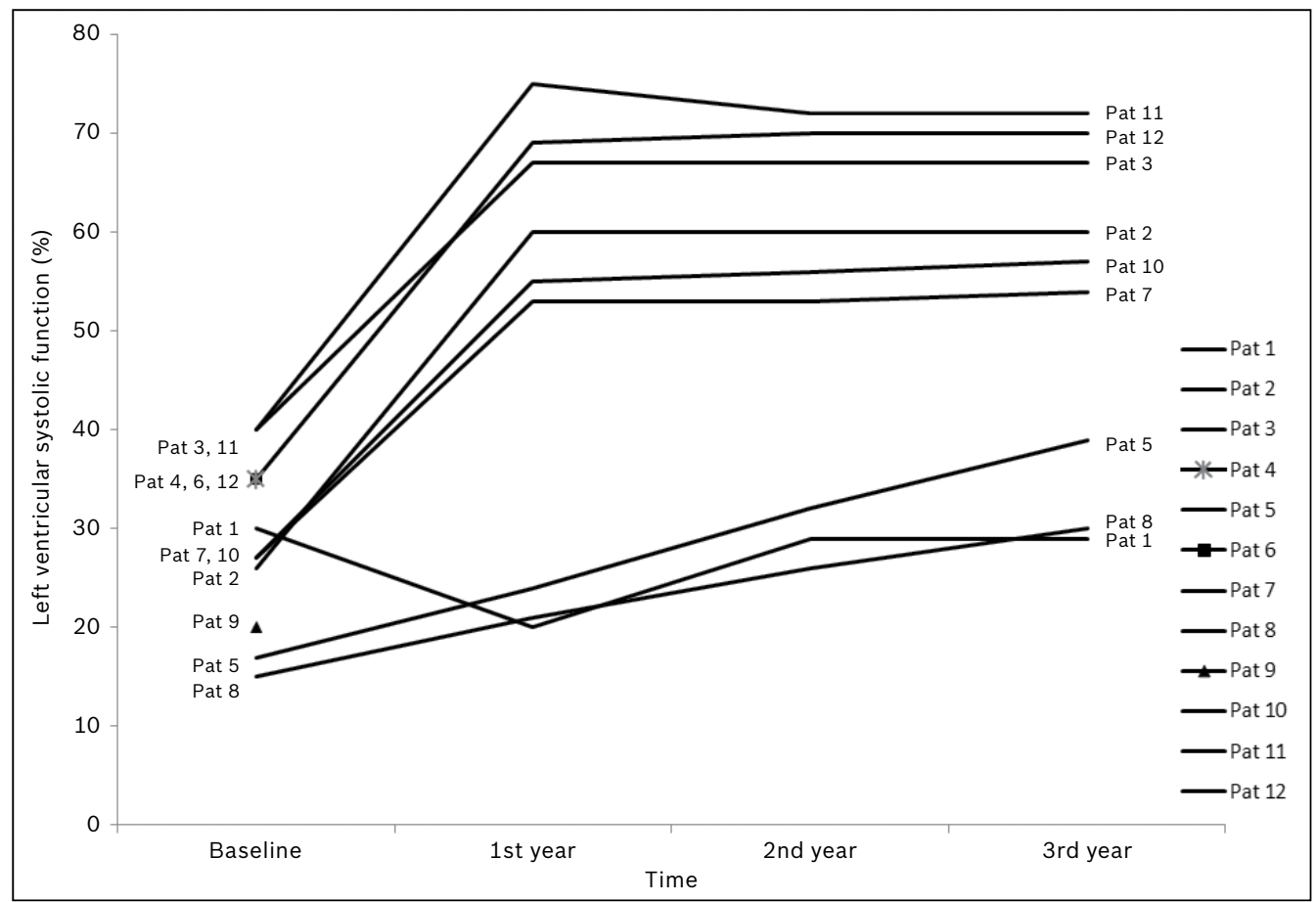

Fig. 1 Graph shows the left ventricular ejection fraction of patients at baseline and during follow-up. Patients 4,6 and 9 did not undergo subsequent echocardiography.

prevalence, presentation and outcome of PPCM among women who gave birth at a major referral centre in Malaysia over a period of ten years are described.

\section{METHODS}

This was a retrospective review of the prevalence of PPCM over a period of ten years between 1 January 2000 and 31 December 2009 at the University Malaya Medical Centre (UMMC), Kuala Lumpur, Malaysia. UMMC is a 1,200-bed teaching hospital that serves the cities of Kuala Lumpur and Petaling Jaya in Malaysia. Kuala Lumpur is the capital city of Malaysia, while Petaling Jaya is a city that originally developed as a satellite township for Kuala Lumpur. The two cities have a combined population of around 3 million. Following approval from the local ethics committee, a retrospective case record analysis was conducted of the medical records of patients in our hospital using the International Classification of Diseases (ICD-10) codes. ${ }^{(12)}$ The keywords used included 'peripartum cardiomyopathy' and 'cardiovascular diseases complicating pregnancy'. Admission records available at the cardiology and obstetric wards were also searched. All medical notes were reviewed and relevant information, including echocardiographic data, was collected. A diagnosis of PPCM was made based on the recommendations of the NHLBI and NIH, as outlined earlier. All patients included in the study fulfilled the diagnostic criteria.

\section{RESULTS}

A total of 12 patients were diagnosed with PPCM at delivery in UMMC during the study period, which saw 48,373 live births recorded at the hospital. The prevalence of PPCM was found to be 2.48 per 100,000 live births in our series. Six patients were ethnically Malay. The mean age of patients at presentation was $32.2 \pm 6.6$ (range 24-43) years. Eight patients were multiparous, while four were primigravidae. Four women had preeclampsia in previous pregnancies and one had chronic hypertension. Nine patients presented with PPCM symptoms during the postpartum period, while three patients were diagnosed during the last month of pregnancy. All patients presented with symptoms of congestive heart failure. All 12 patients had dyspnoea, and nine patients showed evidence of leg oedema. All except one patient delivered via Caesarean section. Three (25\%) women had twin pregnancies.

All patients underwent transthoracic echocardiography on presentation. The mean LVEF was $28.9 \% \pm 8.5 \%$ (range 15\%-40\%). After the index event, six of the nine patients who underwent subsequent echocardiography during follow-up at one year regained normal LVEFs (LVEF > 50\%). Three patients had persistently poor LVEF. Two patients were lost to follow-up. The median follow-up duration for the remaining patients was 77 months. The LVEFs of the patients are presented in Fig. 1, which shows that most of the patients improved during the first year of follow-up. The LVEF remained the same for the remaining follow-up period for most patients after the first year of follow-up.

Electrocardiography and cardiac markers (creatine kinase and troponins) did not show abnormal characteristics. However, chest radiographs were abnormal in $41.7 \%$ of the patients. The abnormalities included cardiomegaly, pleural effusion and pulmonary oedema. Two patients required ventilation during illness. One ventilated patient, who died as a result of disseminated intravascular coagulopathy, required inotropic support and intraaortic balloon pump insertion. One of the patients, a 29-year-old 
Table I. Clinical characteristics and outcomes of the patients diagnosed with peripartum cardiomyopathy $(\mathrm{n}=12)$.

\begin{tabular}{|c|c|c|c|c|c|c|c|}
\hline $\begin{array}{l}\text { Patient } \\
\text { no. }\end{array}$ & $\begin{array}{l}\text { Ethnicity/age at } \\
\text { diagnosis (yrs) }\end{array}$ & $\begin{array}{l}\text { Year of } \\
\text { diagnosis }\end{array}$ & $\begin{array}{l}\text { Baseline LVEF at } \\
\text { diagnosis (\%) }\end{array}$ & $\begin{array}{l}\text { Previous } \\
\text { preeclampsia/ } \\
\text { hypertension }\end{array}$ & $\begin{array}{l}\text { Twin } \\
\text { pregnancy }\end{array}$ & $\begin{array}{l}\text { LVEF at one-year } \\
\text { follow-up (\%) }\end{array}$ & $\begin{array}{l}\text { Maternal } \\
\text { outcome* }\end{array}$ \\
\hline 1 & Malay/38 & 2003 & 30 & No & No & 29 & Alive \\
\hline 2 & Chinese/27 & 2003 & 26 & No & No & 60 & Alive \\
\hline 3 & Chinese/31 & 2002 & 40 & Yes & No & 67 & Alive \\
\hline 4 & Malay/42 & 2008 & 35 & No & No & NA & Alive \\
\hline 5 & Malay/29 & 2002 & 17 & Yes & No & 35 & Alive \\
\hline 6 & Malay/37 & 2003 & 35 & No & No & NA & Dead \\
\hline 7 & Malay/27 & 2004 & 27 & Yes & Yes & 53 & Alive \\
\hline 8 & Chinese/35 & 2007 & 20 & No & No & 30 & Alive \\
\hline 9 & Others/43 & 2003 & 20 & No & No & NA & Alive \\
\hline 11 & Indian/28 & 2007 & 40 & No & No & 72 & Alive \\
\hline 12 & Indian/24 & 2002 & 35 & Yes & Yes & 61 & Alive \\
\hline
\end{tabular}

*As of 31 December 2009.

LVEF: left ventricular ejection fraction; NA: not available

woman (gravida 2, para 1) presented with heart failure, with frequent sustained ventricular tachycardia that did not respond to standard medical therapy. An internal cardiac defibrillator was implanted without threshold testing at 26 weeks of pregnancy for this patient. She underwent Caesarean section at 32 weeks of pregnancy and did not receive any defibrillation after delivery. Table I summarises the characteristics of the patients in our series.

Live births were recorded for all the women with PPCM. Three newborns had poor Apgar scores, for whom the scores at one minute and after five minutes were $<4$. All three newborns were admitted to the special care nursery, and one newborn who had complications due to respiratory distress syndrome required prolonged stay in the nursery for 38 days. No congenital anomalies were reported in the newborns, and all were subsequently discharged well.

Three women continued to have poor LVEF at the one-year follow-up subsequent to discharge and were treated with standard heart failure medications. These medications included diuretics, angiotensin-converting enzyme inhibitor (ACE-I) or angiotensin receptor antagonist. Only one of these patients could not tolerate $\beta$-blockers. The six patients who had regained normal LVEF on echocardiography during follow-up at one year were also continued on heart failure medications. All patients were on ACE-I or angiotensin receptor antagonist, and only two patients were on $\beta$-blockers. None of the patients were given antiplatelet or anticoagulation therapy.

Two women subsequently became pregnant despite being advised against it. Both patients had regained normal left ventricular function after the index PPCM pregnancy prior to the subsequent pregnancy. Pregnancy was terminated for one patient at seven weeks gestation following counselling. The other patient delivered uneventfully without complications at full term by elective lower segment Caesarean section, and the newborn had normal Apgar scores.

\section{DISCUSSION}

The prevalence of PPCM at our centre, which was 2.48 per 100,000 (1 in 40,322) live births, was low compared to many countries, where studies have reported incidences that vary from 0.88 per 100,000 live births in the United States ${ }^{(13)}$ to 250 per 100,000 live births in Haiti. ${ }^{(14)}$ The reported prevalence of PPCM in India was 1 per 1,374 live births or nearly 72 per 100,000 live births, according to Pandit et al. ${ }^{(15)}$ Although the reason behind such variations in the reported prevalence of PPCM from various countries is not yet fully understood, it is possible that several contributory factors, including differences in identification protocols, diagnostic criteria and study populations, might be behind it. As our hospital also serves as a tertiary referral centre, it is possible that the prevalence rate in our cohort was biased by referral patterns. Nevertheless, this study represents the first long-term series of PPCM in Asia.

Treatment of PPCM is along the lines of other forms of congestive heart failure in pregnancy. However, a recent study by Yamac et al reported that a $16-\mathrm{kDa}$ fragment of prolactin may induce myocardial damage, ${ }^{(16)}$ and this has proffered a novel treatment option for PPCM that aims to block prolactin with bromocriptine. Although some studies have successfully used this therapy, ${ }^{(17,18)}$ various others have also advised caution when using bromocriptine for the treatment of PPCM. ${ }^{(19,20)}$ For instance, Hopp et al reported acute myocardial infarction in a healthy woman using bromocriptine for lactate suppression. ${ }^{(21)}$ It should therefore be borne in mind that it would be difficult to conduct large prospective clinical trials to guide treatment in this group of patients. This is especially true for patients who later recover their left ventricular function subsequent to the index PPCM.

Generally, the outcome appears to be better in patients who recover left ventricular function. In our series, of the two women who had normal recovery of left ventricular function during follow-up and became pregnant subsequently, one elected for termination of pregnancy at seven weeks while the other went on 
to deliver at term without any complication. However, this study was not without its limitations. The data presented here is only representative of the study population. Diagnosis was made solely based on the ICD-10 coding of PPCM, and this may have given rise to some bias. Additionally, the study population was small and sourced from a single referral centre.

In conclusion, PPCM was uncommon in this series from Malaysia. It may be associated with twin pregnancies, as seen in $25 \%$ of the women diagnosed with PPCM in this study. Patient outcome was favourable, with $66.7 \%$ of women diagnosed with PPCM recovering normal left ventricular function. The mortality rate was low at $8.3 \%$.

\section{REFERENCES}

1. Gouley BA, McMillan TM, Bellet S. Idiopathic myocardial degeneration associated with pregnancy and especially the puerperium. Am J Med Sci 1937; 194:185-99.

2. Cunningham FG, Pritchard JA, Hankins GD, et al. Peripartum heart failure: idiopathic cardiomyopathy or compounding cardiovascular events? Obstet Gynecol 1986; 67:157-68.

3. Desai D, Moodley J, Naidoo D. Peripartum cardiomyopathy: experiences at King Edward VIII Hospital, Durban, South Africa and a review of the literature. Trop Doct 1995; 25:118-23.

4. Chee KH, Azman W. Prevalence and outcome of peripartum cardiomyopathy in Malaysia. Int J Clin Pract 2009; 63:722-5.

5. Demakis JG, Rahimtoola SH. Peripartum cardiomyopathy. Circulation 1971; 44:964-8.

6. Pearson GD, Veille JC, Rahimtoola S, et al. Peripartum cardiomyopathy: National Heart, Lung, and Blood Institute and Office of Rare Diseases (National Institutes of Health) workshop recommendations and review. JAMA 2000; 283:1183-8.

7. Safirstein JG, Ro AS, Grandhi S, et al. Predictors of left ventricular recovery in a cohort of peripartum cardiomyopathy patients recruited via the internet. Int J Cardiol 2012; 154:27-31.

8. Elkayam U, Tummala PP, Rao K, et al. Maternal and fetal outcomes of subsequent pregnancies in women with peripartum cardiomyopathy. $\mathrm{N}$ Engl J Med 2001; 344:1567-71.

9. Fett JD, Fristoe KL, Welsh SN. Risk of heart failure relapse in subsequent pregnancy among peripartum cardiomyopathy mothers. Int J Gynaecol Obstet 2010; 109:34-6.

10. O'Connell JB, Costanzo-Nordin MR, Subramanian R, et al. Peripartum cardiomyopathy: clinical, hemodynamic, histologic and prognostic characteristics. J Am Coll Cardiol 1986; 8:52-6.

11. Lampert MB, Lang RM. Peripartum cardiomyopathy. Am Heart J 1995; 130:860-70.

12. World Health Organization. ICD-10 Classifications of Mental and Behavioural Disorder: Clinical Descriptions and Diagnostic Guidelines. Geneva: WHO, 1992.

13. Whitehead SJ, Berg CJ, Chang J. Pregnancy-related mortality due to cardiomyopathy: United States, 1991-1997. Obstet Gynecol 2003; 102:1326-31.

14. Fett JD. Peripartum cardiomyopathy. Insights from Haiti regarding a disease of unknown etiology. Minn Med 2002; 85:46-8.

15. Pandit V, Shetty S, Kumar A, Sagir A. Incidence and outcome of peripartum cardiomyopathy from a tertiary hospital in South India. Trop Doct 2009; 39:168-9.

16. Yamac H, Bultmann I, Sliwa K, Hilfiker-Kleiner D. Prolactin: a new therapeutic target in peripartum cardiomyopathy. Heart 2010; 96:1352-7.

17. Habedank D, Kühnle Y, Elgeti T, et al. Recovery from peripartum cardiomyopathy after treatment with bromocriptine. Eur J Heart Fail 2008; 10:1149-51.

18. Jahns BG, Stein W, Hilfiker-Kleiner D, Pieske B, Emons G. Peripartum cardiomyopathy--a new treatment option by inhibition of prolactin secretion. Am J Obstet Gynecol 2008; 199:e5-6.

19. Iffy L, O'Donnell J, Correia J, Hopp L. Severe cardiac dysrhythmia in patients using bromocriptine postpartum. Am J Ther 1998; 5:111-5.

20. Fett JD. Caution in the use of bromocriptine in peripartum cardiomyopathy. J Am Coll Cardiol 2008; 51:2083; author reply 2083-4.

21. Hopp L, Weisse AB, Iffy L. Acute myocardial infarction in a healthy mother using bromocriptine for milk suppression. Can J Cardiol 1996; 12:415-8.

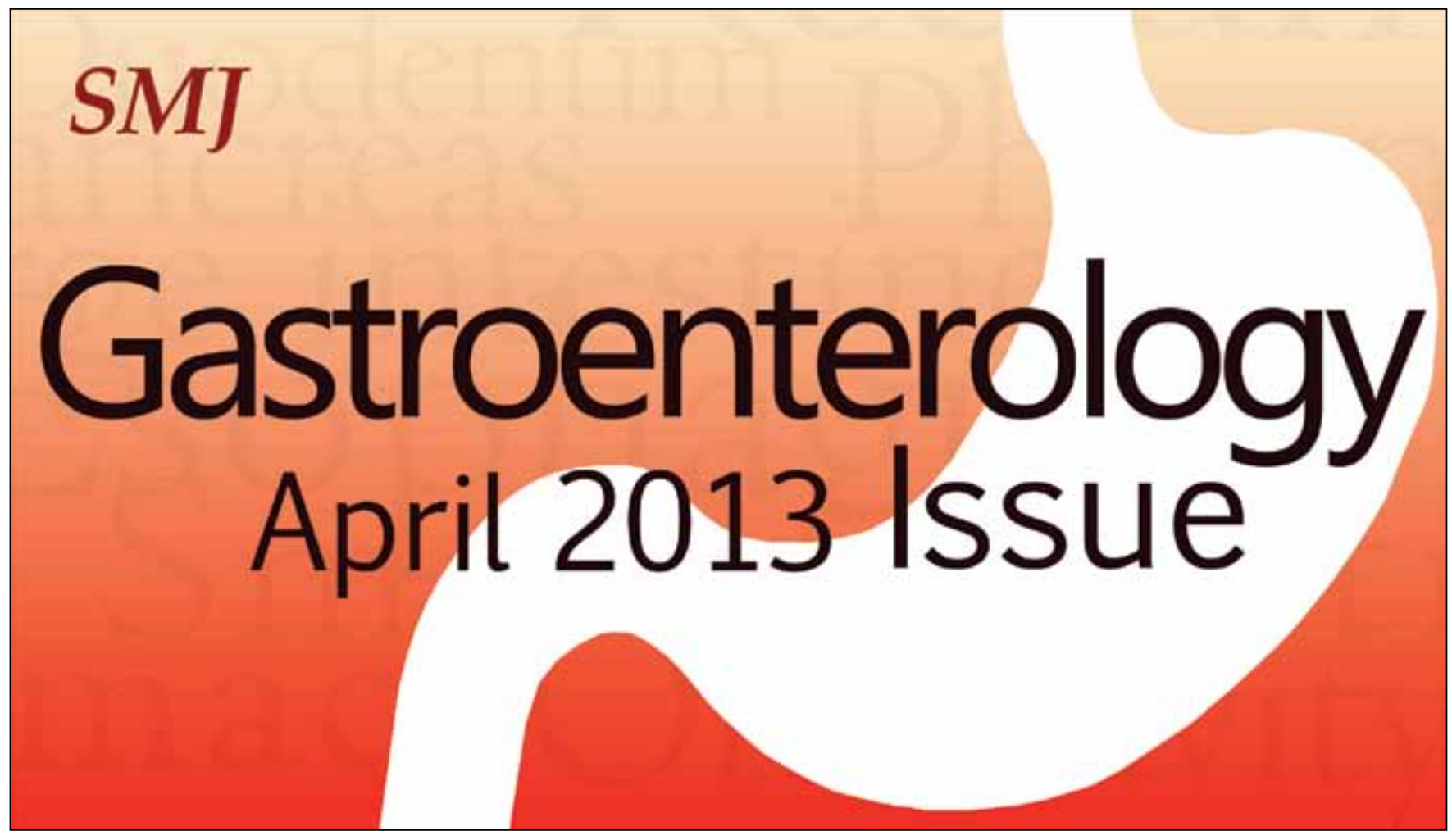

